\title{
Author Correction: Nitrate-NRT1.1B-SPX4 cascade integrates nitrogen and phosphorus signalling networks in plants
}

Bin Hu DiD, Zhimin Jiang, Wei Wang, Yahong Qiu, Zhihua Zhang, Yongqiang Liu, Aifu Li, Xiaokai Gao, Linchuan Liu, Yangwen Qian, Xiahe Huang, Feifei Yu, Sai Kang, Yiqin Wang, Junpeng Xie, Shouyun Cao, Lianhe Zhang, Yingchun Wang, Qi Xie, Stanislav Kopriva (iD and Chengcai Chu (iD)

Correction to: Nature Plants https://doi.org/10.1038/s41477-019-0384-1, published online 25 March 2019.

In the version of this Article originally published, the authors mistakenly presented incorrect images in Fig. $4 \mathrm{~g}$ and h; this has now been amended and the correct Fig. 4g,h panels are shown below. Additionally, the authors mistakenly duplicated images from Fig. $3 \mathrm{~d}$ in Supplementary Fig. 5a; this has now been amended and the correct Supplementary Fig. 5a is shown below.

g

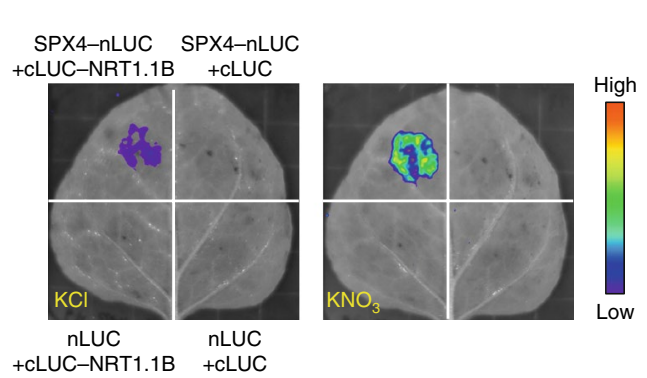

h

$$
\begin{array}{cc}
\text { SPX4-nLUC } & \text { SPX4-nLUC+CLUC } \\
\text { +CLUC-NRT1.1B } & - \text { NRT1.1B } \\
\text { H362A } & -20
\end{array}
$$

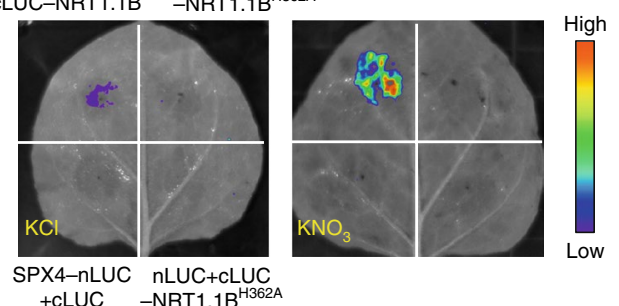

g

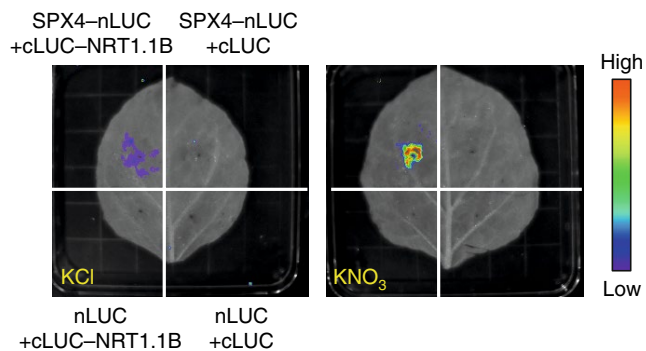

h $\begin{array}{cc}\text { SPX4-nLUC } & \text { SPX4-nLUC+CLUC } \\ + \text { cLUC-NRT1.1B } & -\mathrm{NRT} 1.1 \mathrm{~B}^{\mathrm{H} 362 \mathrm{~A}}\end{array}$

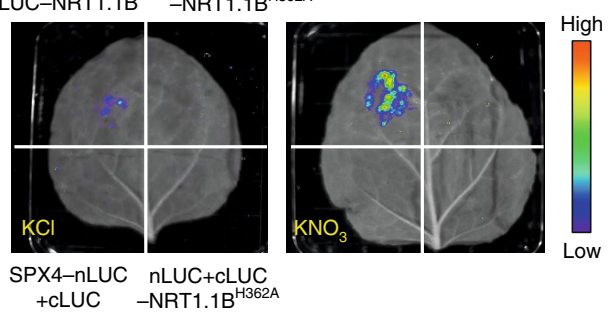

Fig. 4 | Original and corrected.

Original

a
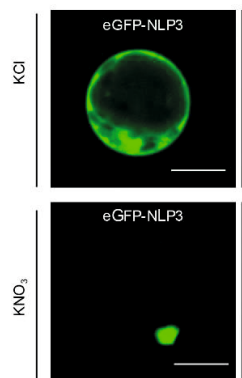

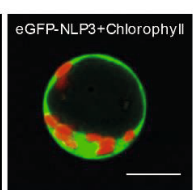

SEP-NLP3+Chlorophyll

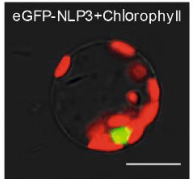

Corrected

a
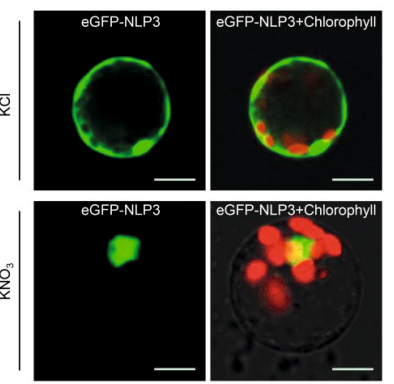

Corrected

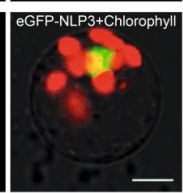

Supplementary Fig. 5a | Original and corrected. 